\title{
A model of spur gears supported by ball bearings
}

\author{
F. Viadero, A. Fernandez del Rincon, R. Sancibrian, \\ P. Garcia Fernandez \& A. de Juan \\ Department Structural and Mechanical Engineering, \\ University of Cantabria, Santander, Spain
}

\begin{abstract}
In this work a model of a 2D spur gear transmission is described for analysis of tooth contact forces and deformations. Assuming the position of each wheel is known, the contact points between gears are obtained taking into account the geometric description of the tooth profiles including profile errors and relief modifications. Then the deformation in each contact point is separated into a global and a local term combining a finite element model and an analytical formulation originating from Hertzian contact theory. The proposed procedure does not need new element meshing for each angular position thus obtaining an important computational advantage. Afterwards, a non-linear system of equations is obtained and solved for each gear position in order to calculate the meshing contact forces. The model can include the possibility of bidirectional single-flank or double-flank action as well as friction forces in the out-of-action line. Once the contact forces are known, it is possible to use the procedure in the calculation of loaded transmission error and meshing stiffness. Furthermore, each gear is supported by ball bearings that are included in the model taking into account their clearance and their variable stiffness due to the change in the number of balls supporting the load. This variable bearing compliance modifies the gear centre distance and as a consequence the transmission error during a turn. Using this methodology a numerical example is presented where the static behaviour of a spur gear transmission is described and analysed. Special attention is focused on the influence of load level on the final loaded transmission error.
\end{abstract}

Keywords: gear, transmission error, bearings, tooth contact, load ratio. 


\section{Introduction}

Gears are one of the most important mechanical components in many advanced machines. Ranging from industry to space, automotive or agricultural equipment, there are a wide range of machines that use gears. This fact justifies the interest in the study of gear dynamics with the aim of design, condition monitoring and vibration and noise control. In this sense it is possible to find works about topics related to gear dynamics such as profile modifications, surface generation kinematics, non-linear interactions, noise, friction, dynamic tooth loads, wear, contact and bending stresses, condition monitoring, efficiency, etc. All of these subjects are also closely related to the so-called Transmission Error (TE), which is defined as "the difference between the position that the output shaft of a drive would occupy if the drive were perfect and the actual position of the output" [1]. There are three main sources of TE: geometry, deflections and dynamics. Although transmission error is a common term used by the gearing community it is possible to add certain names depending on the source that produces the final TE. Thus, it is possible to distinguish between manufacturing, kinematic (sometimes confused with manufacturing), static and dynamic TE. In this work we are interested in the static transmission error also known as Loaded Transmission Error (LTE) as this could be used as an external input excitation for dynamic analysis [2] or as a measure directly related to the noise level of a certain gear transmission.

There is also one other quantity of interest, the meshing stiffness, which governs the dynamic behaviour of geared systems acting as a parametric excitation of the gear pair. The periodic change of the number of contacting teeth pairs is one of the most important phenomena involved in gear dynamics. There are several approaches to include it in the dynamic models from the simple average throughout a meshing cycle to more accurate formulations taking into account the stiffness variation along the tooth profile for each pair $[3,4]$. Nevertheless, some aspects are normally neglected such as the elastic coupling between successive teeth under load, the non-linear variation with the load level or the influence of the support deflection in the final meshing stiffness. Many available dynamic models use a simple formulation for the gear stiffness as their interest is focused on dynamics neglecting all of the terms that could be very useful in certain cases. Special attention should be paid to the case of condition monitoring where a good description of the dynamic forces is crucial for good prediction of machinery condition [5]. The most accurate works [6,7] use the finite element method combined with contact algorithms for evaluating the forces in meshing teeth. Nevertheless, a new meshing is necessary for each angular position, thus making it a high consumption computational task. There are some proposals to avoid this such as the application of Artificial Neural Networks [8] or the combination of finite elements and analytical formulations for the contacts $[9,10]$.

In this work a new model for analysis of contact forces between gear pairs including the interaction with rolling bearings is presented. The gear model is based on the formulation proposed in [9], while bearings include clearances and 
the non linearity due to the changing number of rolling elements supporting the load, following the approach proposed in [11]. Bearing clearances and variable stiffness modify the operation distance between gear centres as well as the pressure angle of the transmission. As a consequence, LTE, meshing stiffness and load ratio should be modified. In this work the proposed model is used only for quasi-static calculations in order to obtain the teeth contact forces and deflections as well as their derived quantities such as LTE, load ratio and meshing stiffness.

\section{Gear contact forces}

The calculation of gear contact forces requires the solution of three different problems; the description of the gear body geometry, the determination of the contact points as a function of the gear position, and the calculation of the contact forces themselves.

The first task will be the definition of the tooth profiles in order to be able to solve the other problems. In this work, the gear generation will be based on a rack-type tool following Litvin's vector approach [12] taking into account the possibility of tool displacements and also undercutting conditions. Furthermore, a rounding profile was added in the tooth tip to handle corner contact.

Contact points and their corresponding separation distances $\left(\boldsymbol{\delta}_{i}\right)$ have been obtained taking advantage of the analytical properties of involute profiles and tip rounding arcs. Positive values for separation distance mean that the points should be in contact and negative values indicate a non-contact condition. Two types of contacting profiles have been considered: involute-involute and involute-circle arc. The number of potential contact points will depend on the contact ratio ( $\varepsilon$ ) and can be expressed as

$$
N=2(\operatorname{Ceil}(\varepsilon)+1)
$$

where $\operatorname{Ceil}(x)$ is a function that rounds $x$ to the nearest integer towards infinity. Contacts on both flanks of any contacting tooth have been considered. In figure 1 the potential contact points for a spur gear pair with a contact ratio between land 2, which means two contact teeth pairs, are shown.

The calculation of gear forces requires a relationship between the forces and displacements of the contact points. This relation should take into account not only the elastic deflection of a pair of teeth in contact but also the local deflection in the vicinity of the load as well as the load sharing between more than one teeth pair. Due to the complexity of gear geometry, which is composed of several parts (involute, fillet, tip rounding), a common procedure for handling this problem is the development of a finite element model for two gears or only a portion of their teeth and applying a type of gap elements in order to simulate the contact $[6,7,13]$. Special attention should be paid to the definition of contact loads and boundary conditions. The resulting load, neglecting frictional forces, should be approximated to the elliptic distribution characteristic of the Hertzian contacts. Furthermore, as the contact width will be affected by the load, a small size meshing in the vicinity of contact points should be provided to be valid from 
low to high load levels. Finally, a different meshing is desired for each position of the contact along the tooth profile. This approach is very time consuming and would not be practical if a dynamic simulation were desired.
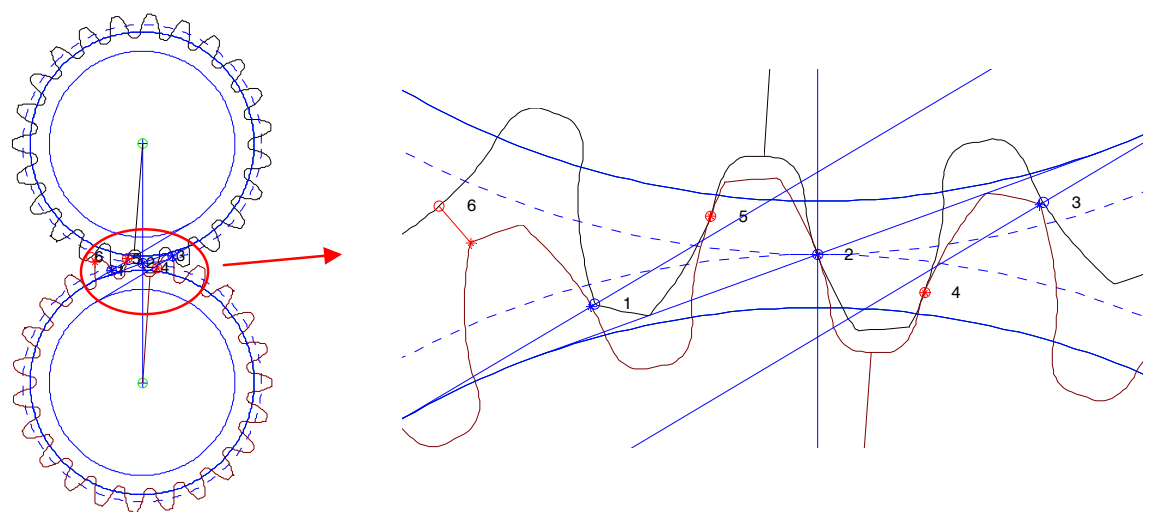

Figure 1: Potential contact points.

Another possibility is to consider the elastic deflections divided in two different contributions: local deflections of Hertzian type in the vicinity of contact points and global deflections, also called structural, that include all the deflections (bending, and shearing) except those due to the local contact.

Local deflections are normally approximated using a non-linear formulation based on Herztian theory or any of its variants. In this work, the local deformation was obtained applying the following expression derived by WeberBanashek for bi-dimensional problems. The deformation between the surface and a line at a depth $\boldsymbol{h}$ is

$$
u_{L}(q)=\frac{2\left(1-v^{2}\right)}{\pi E} q\left[\operatorname{Ln}\left(\frac{h}{L}+\sqrt{1+\left(\frac{h}{L}\right)^{2}}\right)-\frac{v}{1-v}\left(\frac{h}{L}\right)^{2}\left(\sqrt{1+\left(\frac{h}{L}\right)^{2}}-1\right)\right]
$$

where $\boldsymbol{q}$ is the load intensity along the thickness and $\boldsymbol{L} \boldsymbol{L}$ is the extension of the elliptic distribution of the pressure around the location of the load, which can be written as

$$
L=\sqrt{\frac{4}{\pi}\left(\frac{1-v_{p}^{2}}{E_{p}}+\frac{1-v_{g}^{2}}{E_{g}}\right) q \rho} ; \quad \rho=\frac{\rho_{p} \rho_{g}}{\rho_{p}+\rho_{g}}
$$

On the other hand, structural deflections have been obtained by means of a plane strain (or plane stress) finite element model presented in figure 2. The model only contains a certain number of teeth depending of the contact ratio of the gear pair. A unitary load is applied in each node in the profile of the tooth located in the vertical position, which will be called loaded active flank, obtaining the displacement in the rest of the nodes of the other teeth both in the right flank but also in the left one in order to apply the procedure in case of two tooth flanks contacts. The displacements obtained will be the flexibility coefficients $\left(\boldsymbol{\beta}_{i, j}\right)$ which represent the displacement of the node $\boldsymbol{j}$ due to a unitary load applied in the node $\boldsymbol{i}$ of the loaded active flank. 


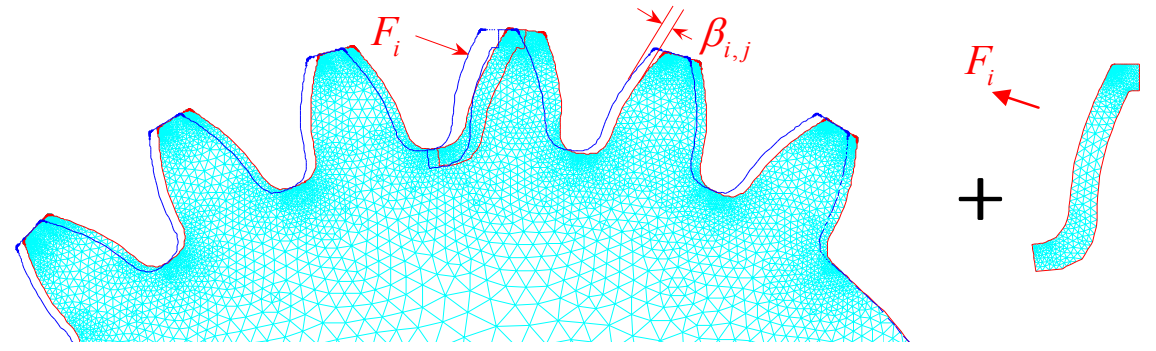

Figure 2: Load and displacements of the finite element model.

This procedure is not valid for the point where the load is applied, as it is a stress concentration point. In this case, a local finite element model with the same meshing as the global one and a depth $\boldsymbol{h}$ is used to correct the displacements in the vicinity of the load point. This model has a unitary load acting in the opposite sense to the one applied in the global model, considering the nodes on the boundary with the global model as fixed. The displacement obtained with this model is removed from the original one avoiding the inaccuracy in the local displacement of the node where the concentrated load was applied. Then, when $\boldsymbol{n}$ contacts take place, the structural displacements $\boldsymbol{u}_{\boldsymbol{S j}}$ of node $\boldsymbol{j}$ will be defined by

$$
u_{S j}=\sum_{i}^{n}\left(\beta_{i, j} F_{i}\right)
$$

The total displacement of node $\boldsymbol{j}$ is obtained by addition of local and structural components for both gears, wheel and pinion, which for $\boldsymbol{n}$ contacts is

$$
u_{T j}=u_{L j}^{p}\left(F_{j}\right)+u_{L j}^{w}\left(F_{j}\right)+u_{L j}^{p}\left(\left\{F_{1}, \ldots, F_{n}\right\}\right)+u_{L j}^{w}\left(\left\{F_{1}, \ldots, F_{n}\right\}\right)
$$

Once the initial separations $\left\{\boldsymbol{\delta}_{i}\right\}$ of the contact points are known as a function of the pinion and wheel centre displacement $\left(\boldsymbol{r}_{p}, \boldsymbol{r}_{w}\right)$, and angular position, the load distribution $\left\{\boldsymbol{F}_{i}\right\}$ of gear teeth can be defined on the basis of the following conditions: compatibility of initial separations and elastic deflections and complementary condition to avoid non realistic negative loads. Taking into account the profile errors for each gear the resulting forces should be obtained from the following non-linear system of equations for $n$ contact points defined by a positive separation distance

$$
\delta_{i}\left(\left\{\vec{r}_{p}, \theta_{p}\right\},\left\{\vec{r}_{w}, \theta_{w}\right\}\right)=u_{T j}\left(\left\{\vec{r}_{p}, \theta_{p}\right\}\left\{\vec{r}_{w}, \theta_{w}\right\}\{F\}\right)+e_{i p}\left(\left\{\vec{r}_{p}, \theta_{p}\right\}\right)+e_{i w}\left(\left\{\vec{r}_{w}, \theta_{w}\right\}\right)
$$

under the condition that $F_{i} \geq 0 ; \quad i=1, \ldots, n$

Here $\boldsymbol{r}_{\boldsymbol{p}}$ and $\boldsymbol{r}_{w}$ are both vectors representing the location for pinion and wheel, $\boldsymbol{\theta}_{\boldsymbol{p}}, \boldsymbol{\theta}_{\boldsymbol{w}}$ are the angular positions for each gear and $\boldsymbol{e}_{\boldsymbol{i}}, \boldsymbol{e}_{\boldsymbol{i} w}$ are the profile errors for gear and wheel corresponding to the contact point $i$.

The geometric separation distance will be used as a first step for calculation of contact forces, considering only those that are positive. Nevertheless, the solution algorithm verifies the deflection in the other contact points looking for a new possible contact. Following this procedure it is possible to calculate the forces and their application direction as a function of gear position. 


\section{Ball bearing contact forces}

Forces in ball bearings involve a similar problem to the gear contact. Again the changing number of contacting elements (see fig. 3) involves a variable stiffness. Furthermore, load increases the period with the maximum number of elements in contact and even the maximun number of contacts themselves. Fortunately, bearing geometry is simpler and the following assumptions can be made:

- Planar movement is considered as in the case of gear forces.

- Inner and outer races are rigidly fixed to the gear shaft and support.

- Only deflections of Hertzian type are considered, neglecting bending and shearing of races and rolling elements.

- The angular separation between rolling elements is constant $\left(\boldsymbol{\theta}_{\boldsymbol{b}}=\mathbf{2 \pi} / \boldsymbol{n}\right)$.

- Rolling elements roll on the surface races without slipping.

The last assumption provides a simple expression to calculate the cage angular position, which controls the angular position for each rolling element, as

$$
\theta_{\text {cage }}=\theta_{\text {shaft }}\left(\frac{r}{r+R}\right)
$$

where $\boldsymbol{R}$ and $\boldsymbol{r}$ are the outer and inner race radii (see fig 3 ).
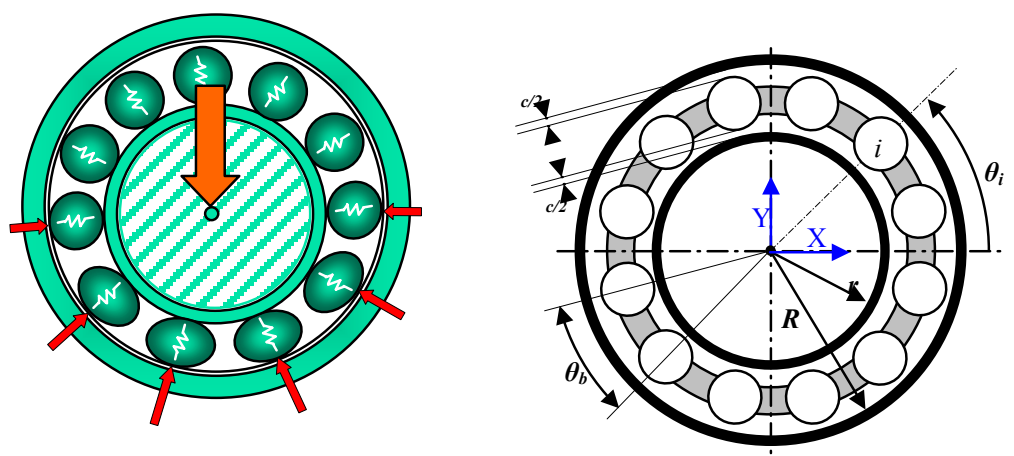

Figure 3: Schema of rolling bearing.

Force deflection relationship for the local Hertzian contact is defined by

$$
F_{\theta i}=k_{B} \delta_{\theta i}^{p} \text { with } \quad p=3 / 2 \text { for ball bearings }
$$

where $\boldsymbol{k}_{\boldsymbol{B}}$ is the contact stiffness, both inner and outer contacts connected in series are considered, and $\boldsymbol{\delta}_{\boldsymbol{\theta} \boldsymbol{i}}$ the radial deformation (geometric overlapping) of the $i^{\text {th }}$ rolling element (located in angular position $\boldsymbol{\theta}_{\boldsymbol{i}}$ ), which will depend on the inner race centre movement $(x, y)$ and the bearing clearance $c$

$$
\delta_{\theta i}^{p}=x \cos \theta_{i}+y \sin \theta_{i}-c
$$

Only positive radial deformations should be considered in the calculation of resultant force as otherwise the ball will not be in contact. Therefore, the resulting force for the $i^{\text {th }}$ rolling element is obtained as 


$$
F_{\theta i}=k_{B} H\left(\delta_{\theta i}\right) \delta_{\theta i}^{p} ; \quad H\left(\delta_{\theta i}\right) \begin{cases}1 & \delta_{\theta i} \geq 0 \\ 0 & \delta_{\theta i}<0\end{cases}
$$

Finally the total force is obtained by summation of the $n$ individual forces from each rolling element. The resultant force, knowing the angular positions $\boldsymbol{\theta}_{i}$, can be expressed by the $\mathrm{x}$ and $\mathrm{y}$ direction components, with the following equations,

$$
\left.\begin{array}{l}
F_{x}=k_{B} \sum_{i=1}^{n} H\left(\delta_{\theta i}\right) \delta_{\theta i}^{p} \cos \theta_{i} \\
F_{y}=k_{B} \sum_{i=1}^{n} H\left(\delta_{\theta i}\right) \delta_{\theta i}^{p} \sin \theta_{i}
\end{array}\right\} ; \quad \text { with } \quad \theta_{i}=\theta_{\text {cage }}+\theta_{b}(i-1)
$$

\section{Application example}

The models described in the previous paragraphs have been applied to a pair of gear wheels with the same number of teeth, each one supported by two identical ball bearings. The main gear parameters are contained in table 1, while the support data bearings are defined in table 2

Table 1: Gear data parameters.

\begin{tabular}{|l|l|}
\hline Parameter & Pinion / Gear \\
\hline Number of teeth $(\mathrm{Z})$ & 23 \\
\hline Module $(\mathrm{m})$ & $3(\mathrm{~mm})$ \\
\hline Modulus of elasticity, E & $210(\mathrm{GPa})$ \\
\hline Poisson's ratio & 0.3 \\
\hline Pressure angle & $20($ degree$)$ \\
\hline Rack addendum & $1.25 \mathrm{~m}$ \\
\hline Rack deddendum & $1 \mathrm{~m}$ \\
\hline Rack tip rounding & $0.25 \mathrm{~m}$ \\
\hline Gear tip rounding & $0.05 \mathrm{~m}$ \\
\hline Gear face width & $15(\mathrm{~mm})$ \\
\hline Gear shaft radius & $9(\mathrm{~mm})$ \\
\hline
\end{tabular}

Table 2: $\quad$ Bearing data parameters.

\begin{tabular}{|l|l|}
\hline Parameter & Value \\
\hline Contact Stiffness $\boldsymbol{k}_{\boldsymbol{B}}$ & $7.05510^{9} \mathrm{~N} / \mathrm{m}^{3 / 2}$ \\
\hline Number of ball bearings $n$ & 9 \\
\hline Radial clearance $c$ & $20(\mu \mathrm{m})$ \\
\hline Outer race radius & $14.13(\mathrm{~mm})$ \\
\hline Inner race radius & $9.37(\mathrm{~mm})$ \\
\hline
\end{tabular}

Before carrying out simulations, taking into account both force models, each one is tested individually to validate it. The bearing model was tested applying an increasing load in the $\mathrm{x}$ direction (corresponding to a torque from -10 to - 
$100 \mathrm{Nm}$ ) obtaining the equilibrium position for a complete turn of the shaft (360 degree). The number of active contacts is shown in figure 4 , while the corresponding orbits are shown in figure 5. It can be observed that a displacement in y direction appears even though there is no force applied in this direction. Moreover displacement in the $y$ direction is greater than the displacement in $\mathrm{x}$ direction. Therefore, when bearings interact with gear forces a variation of the gear centre position should be expected and therefore a modification of the operation distance and pressure angle.

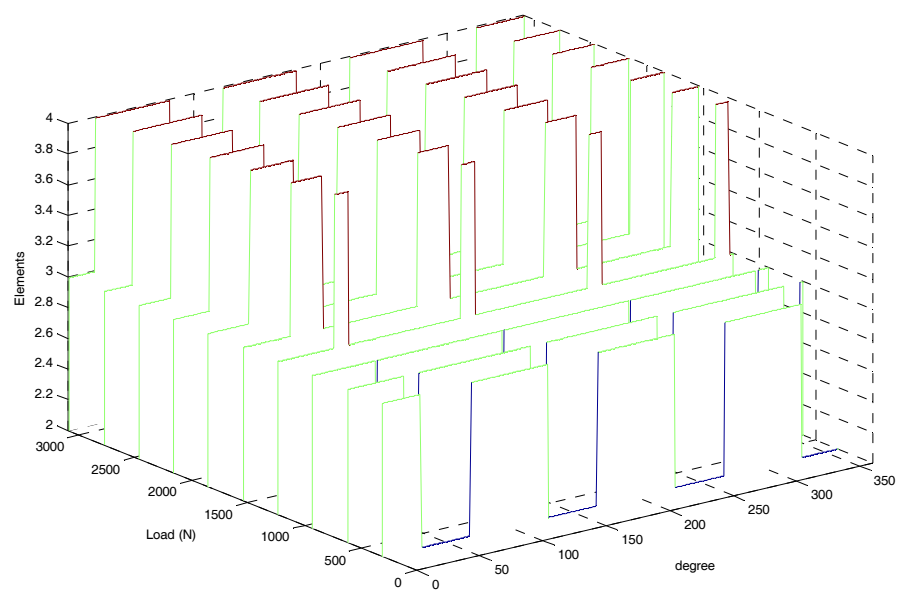

Figure 4: Number of active elements.

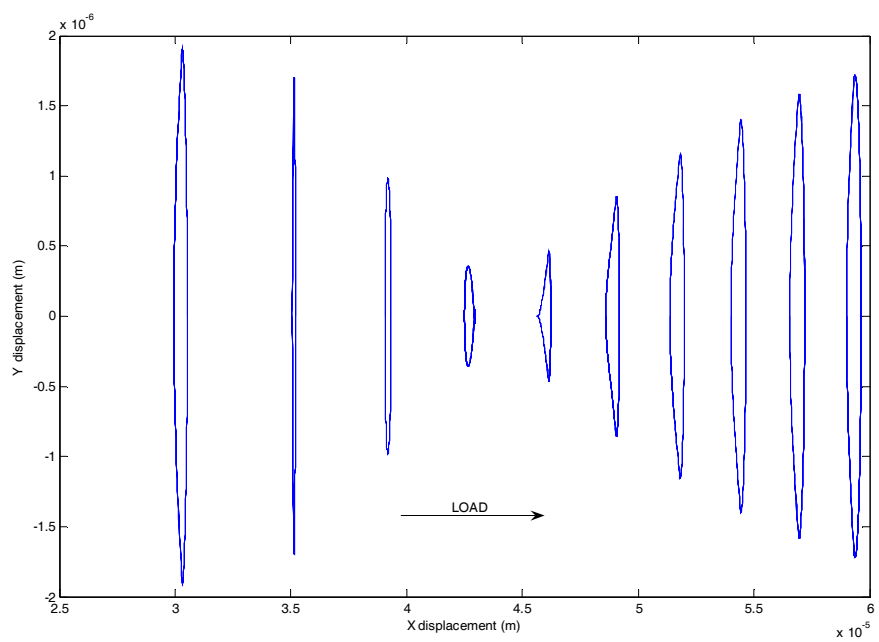

Figure 5: Orbits for several load values. 
The gear model was also tested taking into account a fixed position for gear centres (rigid support) neglecting profile errors. Then an increasing torque (from 10 to $100 \mathrm{Nm}$ ) was applied and the turn angle necessary to obtain the desired torque that will be the LTE was calculated for several angular positions and the torsional stiffness was deduced as in reference [6].

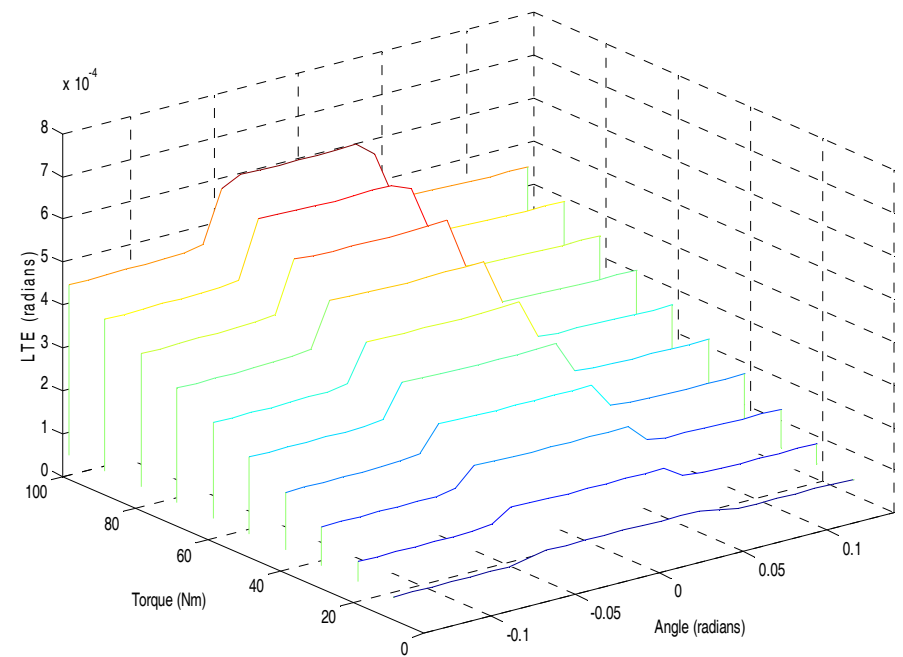

Figure 6: LTE (radians) for several load values (rigid support).

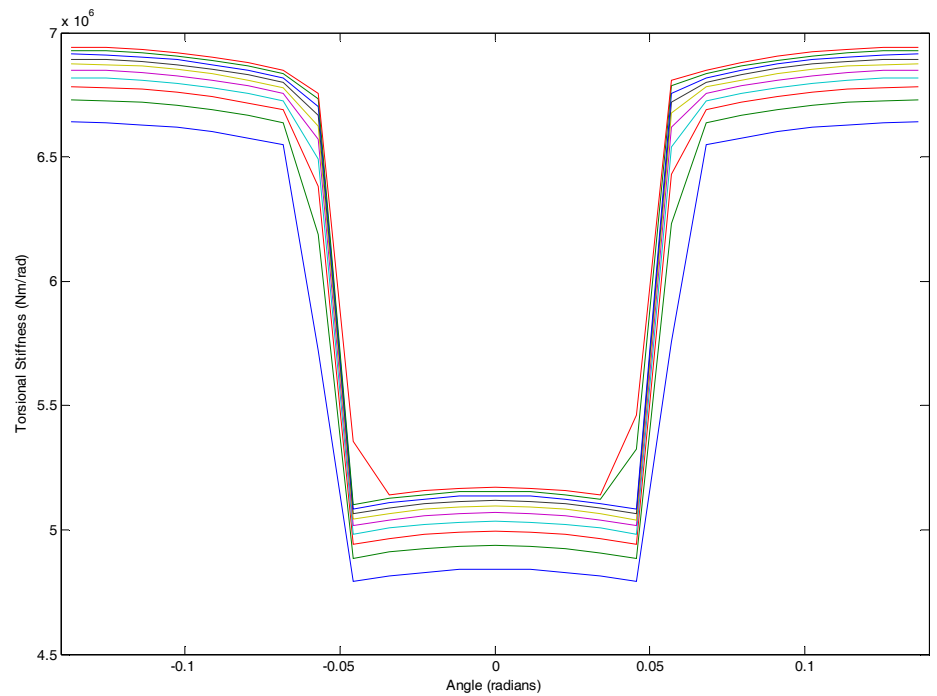

Figure 7: Torsional Stiffness for several load values (rigid support). 
Both magnitudes are shown in figure 6 and figure 7 for several torque levels. Reference angular position corresponds to the contact in the primitive point without any clearance. It is clear that the torque increases the period of double contact (higher stiffness in figure 7) and the corresponding transmission error.

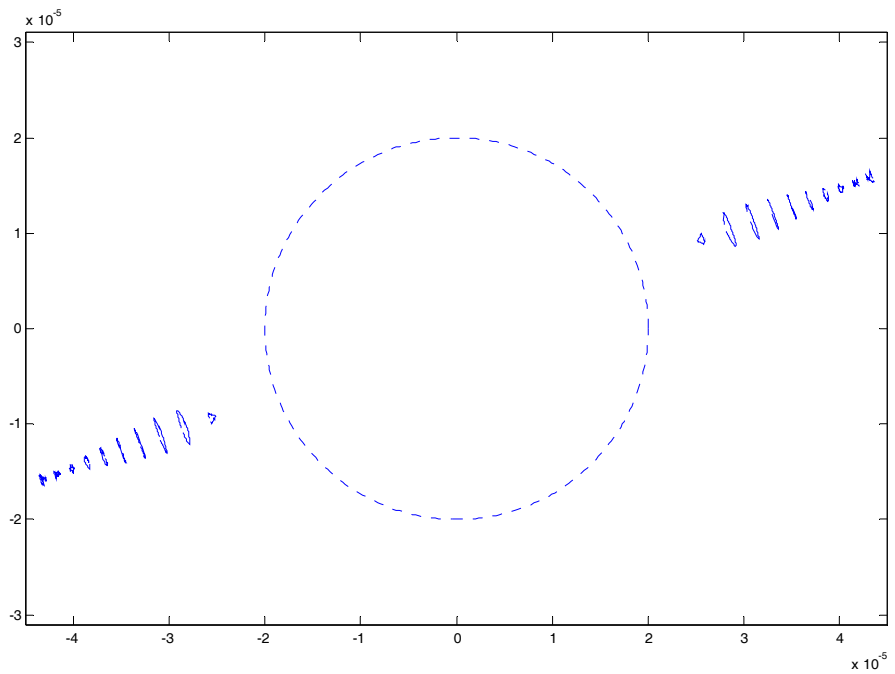

Figure 8: $\quad$ Orbits for several load values with support flexibility.

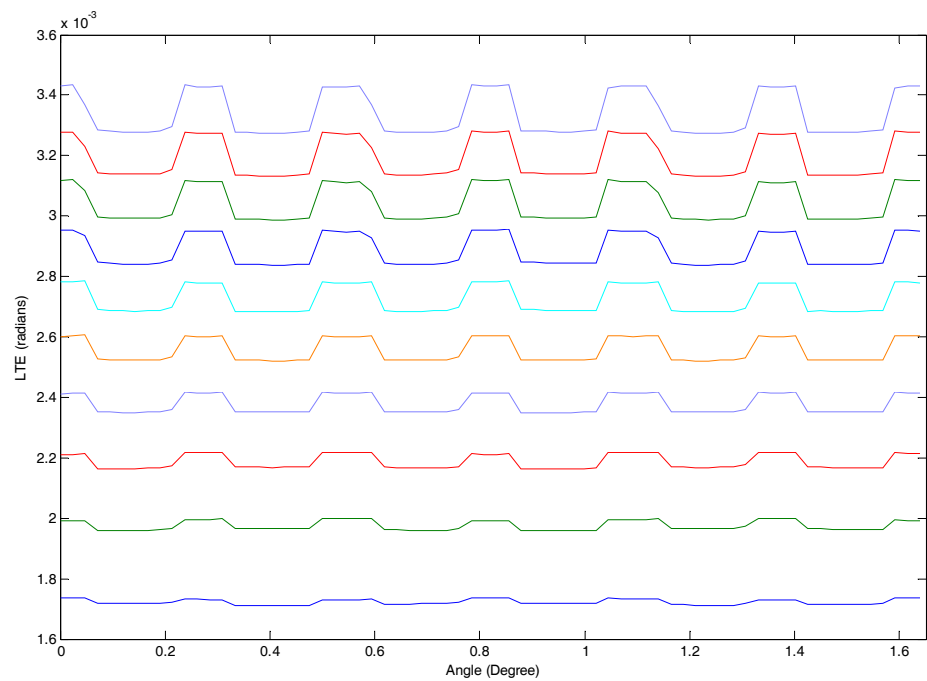

Figure 9: LTE (radians) for several load values with support flexibility. 
Finally the whole model was tested with the same torque levels. This time the interaction between gears and bearings is recorded as can be seen in figure 8 where the orbits for each gear centre are shown. Figure 9 shows the resulting LTE corresponding to the orbits presented in figure 8 , assuming a flexible support due the bearing flexibility. In spite of the fact that, in figure 9 it is not appreciated due to the scale, the model is able to predict an oscillation wave in the resulting LTE due to the bearing's variable compliance. It should also be noted that the LTE shown in the figure 9 should be corrected removing the angular clearance that appears as a consequence of the variable position of gears.

\section{Conclusions}

A quasi-static model for the study of interactions between gears and ball bearings is presented. The main features of the model developed are the approximation of the gear contact by decomposition of local and global deformations and the inclusion of bearing variable compliance with the angular position. An application example was presented where the interaction between elements can be observed taking into account the effect of the load, obtaining several parameters representative of the system behaviour such as the LTE, centre orbits and the number of active contacts in bearings and gears.

In this work some quasi-static analyses were presented, nevertheless one of the aims of this model was its application to the dynamic analysis of gear transmission. Taking into account this fact, special attention was paid to achieving a good compromise between accuracy and computational load.

\section{Acknowledgements}

This paper has been developed in the framework of the Projects DPI2003-1845 and DPI2006-14348 funded by the Spanish Ministry of Science and Technology.

\section{References}

[1] J. Dereck Smith, Gear Noise and Vibration, Marcel Dekker, Inc, 1999.

[2] P. Velex, M. Ajmi, On the modelling of excitations in geared systems by transmission errors, Journal of Sound and Vibration, 290(3-5), pp. 882909, 2006.

[3] J. H. Kuang, Y. T. Yang, An estimate of mesh stiffness and load sharing ratio of a spur gear pair, Proceedings of the ASME International Power Transmission and Gearing Conference 1992.

[4] Y. Cai, T. Hayashi, The linear approximated equation of vibration of a pair of spur gears (theory and experiment), Transactions of the ASME, Journal of Mechanical Design, 116, pp. 558-564, 1994.

[5] A. Parey, M. El Badaoui, F. Guillet, N. Tandon, Dynamic modelling of spur gear pair and application of empirical mode decomposition-based statistical analysis for early detection of localized tooth defect, Journal of Sound and Vibration, 294(3), pp. 547-561, 2006. 
[6] Ian Howard, Shengxiang Jia, Jiande Wang, The dynamic modelling of a spur gear in mesh including friction and a crack, Mechanical Systems and Signal Processing, 15(5), pp. 831-853, 2001.

[7] Shengxiang Jia, Ian Howard, Comparison of localised spalling and crack damage from dynamic modelling of spur gear vibrations, Mechanical Systems and Signal Processing, 20(2), pp. 332-349, 2006.

[8] L. D. MacLennan, An analytical method to determine the influence of shape deviation on load distribution and mesh stiffness for spur gears, Journal of Mechanical Science Part C, 216, pp. 1005-1016, 2002.

[9] A. Andersson, L. Vedmar, A method to determine dynamic loads on spur gear teeth and on bearings, Journal of Sound and Vibration, 267(5), pp. 1065-1084, 2003.

[10] R.G. Parker, S. M. Vijayakar, T.B. Imajo, Non-linear dynamic response of a spur gear pair: modelling and experimental comparisons, Journal of Sound and Vibration 237 (3), pp.435-455, 2000.

[11] M. Tiwari, K. Gupta, O. Prakash, Dynamic response of an unbalanced rotor supported on ball bearings, Journal of Sound and Vibration, 238(5), pp. 757-779, 2000.

[12] F.L. Litvin and A. Fuentes, Gear Geometry and Applied Theory, Cambridge University Press, 2004.

[13] G. Bonori, A. O. Andrisano, F. Pellicano, Stiffness Evaluation and Vibration in a tractor gear, Proceedings of ASME International Mechanical Engineering Congress and Exposition (IMECE) 2004. 